\title{
Aluminum-Copper-Lithium Alloy 2050 Developed for Medium to Thick Plate
}

\author{
Ph. Lequeu, K.P. Smith, and A. Daniélou
}

(Submitted May 20, 2009; in revised form September 1, 2009)

\begin{abstract}
This article discusses Al-Cu-Li 2050 alloy developed, qualified, and produced by Alcan Aerospace as plates. AA2050 alloy offers a low density high corrosion resistant alternative to incumbent medium to thick plate alloys like 7050-T7451, and to thin plate alloys like 2024 or higher damage tolerant versions. The fundamentals behind the choice of the Al-Cu-Li chemistry are highlighted, as well as the property balance generated in a wide range of thickness from 12 to $127 \mathrm{~mm}(0.5$ to $5 \mathrm{in}$.). Manufacturing behavior of the alloy when machining or friction stir welding is also discussed in detail. Finally, it is shown that the appropriate recycling investments and logistics permit the use of 2050 alloy at an acceptable level of extra cost per kilo saved, when weight benefits are taken into account.
\end{abstract}

Keywords aluminum, aerospace, mechanical testing

\section{Introduction}

Aluminum alloys containing lithium are well known for their benefits in reducing density and increasing Young's modulus, proportionally to their Li content.

High Li containing chemistries developed in the 1980's (8090, 8091, 2090, and 2091) exhibited 7 to $10 \%$ density benefit versus baseline alloys of similar strength, but received limited commercial success due to a number of technical problems, which include poor corrosion resistance, low toughness, and high anisotropy.

In the 1990's alloys with high copper content (2195, 2x96, $2 \times 97$, and 2098) were developed and received significant commercial success mostly in the US, on programs like the External Super Light Weight Tank of the Space Shuttle or the F16 fighter, replacing incumbent alloys 2x24 and 2219, in thin tank and fuselage skins, bulkheads or spars.

Design of the most recent commercial airframes like B777, A340-600, and A380 has required the use of more advanced high strength and high damage tolerant alloys, like 7055, 7x49, $2524,2024 \mathrm{~A}, 2027,7 \times 40$, or 7085 .

This article discusses the rationale behind the choice of the chemistry alloy AA2050, and highlights the property balance obtained in the range of thickness 12 to $127 \mathrm{~mm}$ versus thin gauge conventional solutions like 2024 and versus the bestselling medium to thick gauge 7050 incumbents.

Ph. Lequeu, Alcan Rhenalu, Issoire, France; K.P. Smith, Alcan Rolled Products, Ravenswood, WV; and A. Daniélou, Alcan CRV, Voreppe, France. Contact e-mail: armelle.danielou@alcan.com.

\section{Alloy Development}

The alloy 2050 was defined for a medium to high strength and high damage tolerance alloy intended to out-perform the property balance of $2 \mathrm{xxx}$ thin plate alloys and 7xxx thick plate alloys.

Depending on $\mathrm{Cu}$ and $\mathrm{Li}$ content, three main hardening precipitates can be found in $\mathrm{Al}-\mathrm{Cu}-\mathrm{Li}$ alloys (for $\mathrm{Cu}$ in the range 2 to $5 \%$ ).

- For low Li contents $(<0.6 \%)$ the non-shearable $\Omega$ $\left(\mathrm{Al}_{2} \mathrm{Cu}\right)$ phase is present.

- For medium Li content $(<1.4-1.5 \%)$ the main hardening phase is $\mathrm{T}_{1}\left(\mathrm{Al}_{2} \mathrm{CuLi}\right)$ (Ref 1). The hardening effect of this phase is very large and it is promoted by the addition of $\mathrm{Cu}$. Its nucleation and growth are favored by the limited addition of $\mathrm{Mg}$ and $\mathrm{Ag}$ and dislocations formed through cold work prior to artificial aging (Ref 2).

- For high $\mathrm{Li}$ contents $(>1.4-1.5 \%) \delta^{\prime}\left(\mathrm{Al}_{3} \mathrm{Li}\right)$ phase also precipitates during artificial aging and contributes to strengthening but to a lesser extent than $\mathrm{T}_{1}$ (Ref 3); its appearance can be detrimental to toughness. Heavy precipitation of this $\delta^{\prime}$ phase is also reported during longthermal exposure (e.g., $1000 \mathrm{~h}$ at $85^{\circ} \mathrm{C}$ ), and may lead to long-term stability issues, if not properly taken into account in the alloy design.

The phases $\theta^{\prime}$ and $\mathrm{S}^{\prime}$ have also been observed (Ref 3 ).

The chemistry and process of 2050 developed by Alcan Aerospace was registered at the Aluminum Association in November 2004, with the optimized chemical composition given in Table 1 below. Alloy density is $2.70 \mathrm{~g} / \mathrm{cm}^{3}\left(0.098 \mathrm{lb} / \mathrm{in}^{3}\right)$.

For this specific chemistry, Li content was set at a maximum value of $1.3 \%$ to avoid any formation of $\delta^{\prime}$ phases detrimental to thermal stability. $\mathrm{Cu}$ content was adjusted to be sufficiently high to achieve the required strength level, and sufficiently far from the solubility limit in order to maximize strength/toughness balance. For this composition, the main hardening phase is $\mathrm{T}_{1}\left(\mathrm{Al}_{2} \mathrm{CuLi}\right)$. The morphology of this phase is plate-like, as illustrated in Fig. 1. The phase $\theta^{\prime}$ is also present, but to a lesser degree. 
$\mathrm{Mn}$ and $\mathrm{Zr}$ help to promote an unrecrystallized structure, through precipitation as dispersoids; quench precipitates can in some cases initiate on these dispersoids at the grain boundaries (see Fig. 2).

Silver is reported in literature to enhance the nucleation of $\mathrm{T}_{1}$ phases (Ref 4, 5) especially in the presence of magnesium (Ref 6). The nucleation for these plate-like particles is indeed difficult, compared to $\delta^{\prime}$ phases. Silver increases the speed of aging kinetics and also the peak hardness.

Thermo-mechanical processing (TMP) conditions were optimized to get the appropriate property balance in the various thickness ranges of interest. One of the processing parameters to be controlled is the amount of stretching prior to aging, which governs the quantity and size of the $\mathrm{T}_{1}$ hardening phases (Ref 7). For 2050 alloy, a 3.5 to $4 \%$ stretch is being used for

\section{Table 1 AA 2050 registered chemistry}

\begin{tabular}{lccccccccc}
\hline Wt.\% & Si & Fe & Cu & Mn & Mg & Zn & Li & Ag & Zr \\
\hline Min & & & 3.2 & 0.20 & 0.20 & & 0.7 & 0.20 & 0.06 \\
Max & 0.08 & 0.10 & 3.9 & 0.50 & 0.6 & 0.25 & 1.3 & 0.7 & 0.14 \\
\hline
\end{tabular}

this purpose, with an associated T84 temper designation. A typical aging kinetic is illustrated in Fig. 3, corresponding to this amount of controlled stretching; smaller elongations at stretching would lead to longer age practices. Aging treatment was selected as leading to a near-peak, slightly under-aged temper; this choice was because alloy is intended to be thermally stable when subject to further (even low temperature) cycles like $1000 \mathrm{~h}$ at $85^{\circ} \mathrm{C}$.

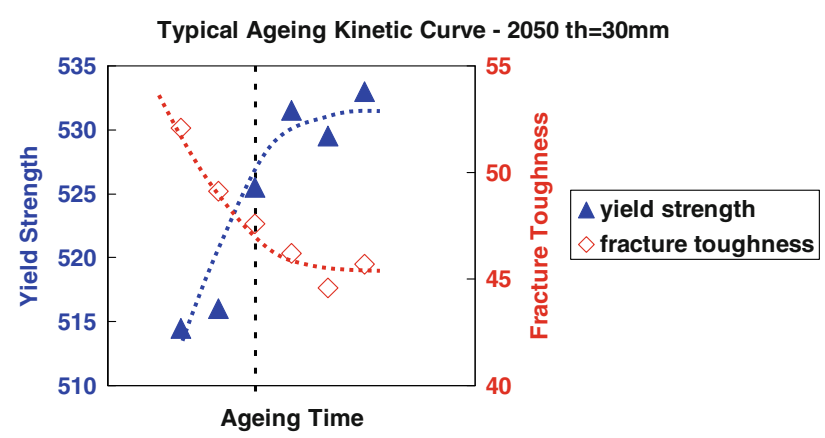

Fig. 3 Aging kinetic of a $30 \mathrm{~mm}$ thick 2050 plate
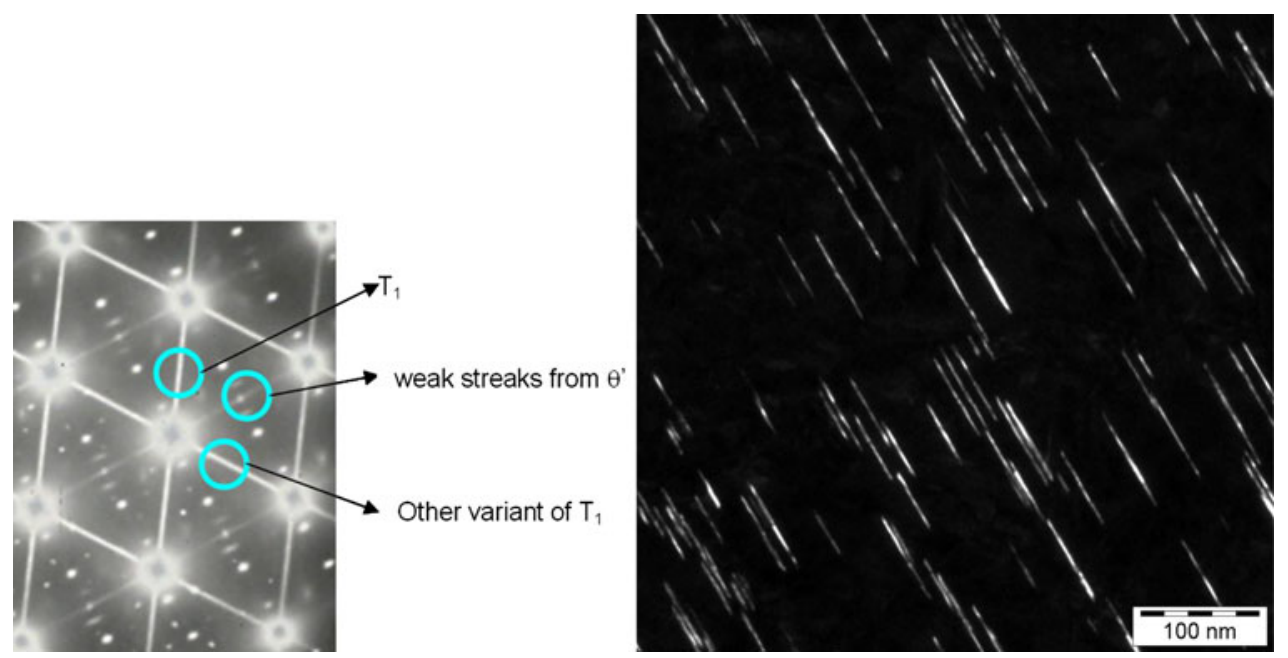

Fig. 1 (Left) Diffraction pattern in [011] Al orientation, alloy 2050-T84. (Right) TEM (dark field) analysis showing precipitation of $\mathrm{T}_{1}$ taken along [011] $\mathrm{Al}$
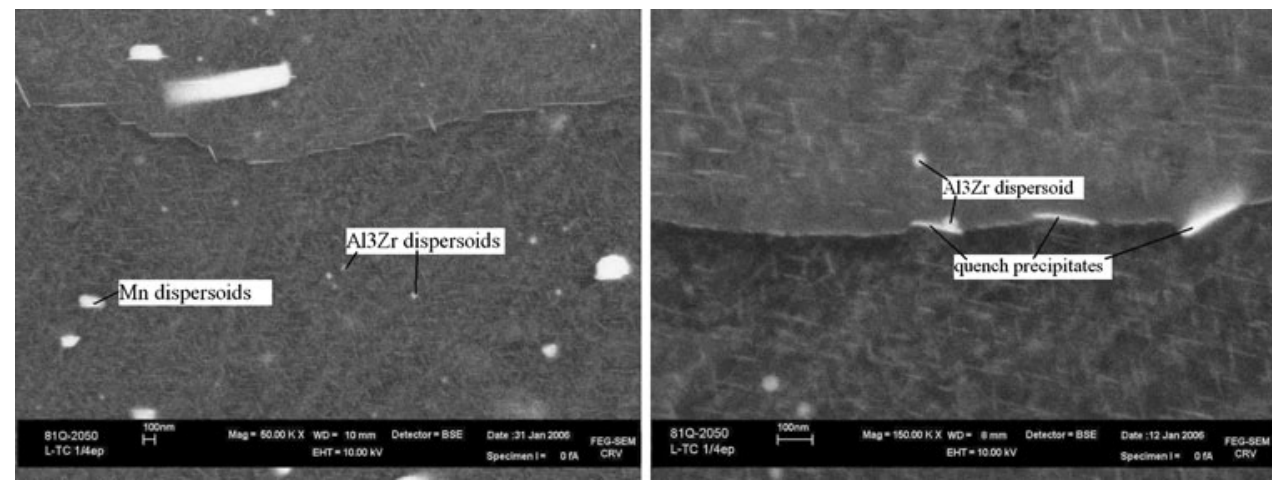

Fig. 2 Dispersoids and quench precipitates, as observed by SEM in 2050-T84 alloy, thick plate 


\section{2050 Plate Properties}

Development of 2050-T84 plate alloy was performed using the Alcan industrial Al-Li casting facility located in Dubuc, Québec, Canada, as well as the industrial equipment of the Issoire (France) and Ravenswood (WV, USA) plants.

More than 30 such ingots were processed with the TMP conditions described above. This was to scan alloy properties within the thickness range to be covered in a first phase, i.e., 12 to $127 \mathrm{~mm}$, and to explore controlled variations of key process parameters in order to anticipate on the industrialization phase. Extensive characterization was performed on every lot, in static, damage tolerance, and corrosion resistance, leading to the coverage of the alloy by AMS4413. Exposure at elevated temperature $\left(1000 \mathrm{~h}\right.$ at $\left.85^{\circ} \mathrm{C}\right)$ shows relatively minor changes in strength and toughness. Low temperature $\left(-65^{\circ} \mathrm{C}\right)$ properties were also investigated, with no detrimental effect over RT measurements. Extension to thickness up to $165 \mathrm{~mm} / 6.5 \mathrm{in}$. is currently being investigated.

Based on all these results, minimum or typical properties can be seen in Table 2, and are compared to incumbent alloy 2024-T351 (for typical thickness of 25-38 mm) and 7050T7451 (for typical thickness of 25-38 and 100-125 mm). For some of the properties, minima have been estimated and are currently being reviewed by the MMPDS (Metallic Materials Properties Development and Standardization) authorities.
It is clearly seen from this table that 2050 alloy, in addition to its density benefit, offers a tremendous improvement over 2024-T351 in static-related properties and corrosion resistance. When compared to incumbent alloy 7050-T7451, 2050-T84 offers an improved (strength, toughness) balance, at 5\% lower density and significantly improved stress corrosion resistance. In addition to these benefits, Fig. 4 demonstrates a fatigue initiation and crack growth benefit of the alloy versus 7050; these improvements can in turn mean additional structural weight benefit of any re-designed part.

\section{2050 Manufacturing Behavior}

2050 plates are intended to be machined and in some cases formed (by mechanical or age forming processes), as every plate made of conventional alloy. In some cases where reduction in buy-to-fly becomes crucial for the overall economics of any part to be converted to 2050, use of friction stir welding (FSW) can also be an interesting option.

Machining behavior of 2050 plates was extensively measured using the barrel method (Ref 8). This method consists in a progressive layer removal of metal by controlled machining of full thickness L \& LT test bars extracted from a plate. Throughthickness stress profiles in both L and LT directions can be obtained in this way; they can also be used as input to FEM

Table 2 Properties of Al-Cu-Li 2050-T84 vs. incumbent 2024-T351 and 7050-T7451

\begin{tabular}{|c|c|c|c|c|c|}
\hline \multirow{2}{*}{$\begin{array}{l}\text { Thickness } \\
\text { Alloy } \\
\text { Basis }\end{array}$} & \multicolumn{3}{|c|}{ 25-38 $\mathrm{mm}$ (1-1.5 in.) } & \multicolumn{2}{|c|}{$100-125 \mathrm{~mm}$ (4-5 in.) } \\
\hline & $\begin{array}{c}\text { 2024-T351 } \\
\text { A-value* }\end{array}$ & $\begin{array}{c}\text { 7050-T7451 } \\
\text { A-value* }\end{array}$ & $\begin{array}{c}2050-T 84 \\
\text { S-value (Est)** }\end{array}$ & $\begin{array}{c}\text { 7050-T7451 } \\
\text { A-value* }\end{array}$ & $\begin{array}{c}\text { 2050-T84 } \\
\text { S-value (Est) }{ }^{* *}\end{array}$ \\
\hline \multicolumn{6}{|l|}{ Ftu, MPa } \\
\hline $\mathrm{L}$ & 427 & 510 & 503 & 490 & 490 \\
\hline LT & 427 & 510 & 510 & 490 & 490 \\
\hline ST & $\ldots$ & $\ldots$ & $\ldots$ & 462 & 476 \\
\hline \multicolumn{6}{|l|}{ Fty, MPa } \\
\hline $\mathrm{L}$ & 324 & 441 & 476 & 421 & 455 \\
\hline LT & 290 & 441 & 462 & 421 & 441 \\
\hline ST & $\ldots$ & $\ldots$ & $\ldots$ & 393 & 407 \\
\hline \multicolumn{6}{|l|}{ Fcy, MPa } \\
\hline $\mathrm{L}$ & 269 & 434 & 470 & 400 & 460 \\
\hline LT & 303 & 455 & 480 & 441 & 475 \\
\hline ST & $\ldots$ & $\ldots$ & $\ldots$ & 434 & 470 \\
\hline Fsu, MPa & 255 & 296 & 290 & 296 & 295 \\
\hline Fbru, MPa, $e / D=1.5$ & 648 & 738 & 735 & 738 & 780 \\
\hline Fbru, $\mathrm{MPa}, e / D=2.0$ & 793 & 965 & 960 & 951 & 1050 \\
\hline \multicolumn{6}{|l|}{$e$, percent } \\
\hline $\mathrm{L}$ & $\ldots$ & 10 & 8 & 9 & 5 \\
\hline LT & 7 & 9 & 6 & 5 & 3 \\
\hline ST & $\cdots$ & $\ldots$ & $\ldots$ & 3 & 1.5 \\
\hline \multicolumn{6}{|l|}{$\mathrm{KIc}, \mathrm{MPa} \sqrt{ } \mathrm{m}$} \\
\hline L-T & $(30)$ & 29 & 36 & 27 & 28 \\
\hline $\mathrm{T}-\mathrm{L}$ & $\ldots$ & 25 & 32 & 24 & 23 \\
\hline S-L & $\ldots$ & $\ldots$ & $\ldots$ & 23 & 23 \\
\hline \multicolumn{6}{|l|}{ Stress corrosion, $\mathrm{MPa}$} \\
\hline LT, 30 days & $>170$ & $\ldots$ & 450 & $\ldots$ & $\ldots$ \\
\hline ST, 30 days & $\cdots$ & $\ldots$ & $\ldots$ & 240 & 310 \\
\hline Exfoliation corrosion & ED & EA & EA & EA & EA \\
\hline Density, g/cm³ ${ }^{3}$ typ. & 2.78 & 2.83 & 2.70 & 2.83 & 2.70 \\
\hline$E$, GPa, Tyр. & 69 & 69 & 76.5 & 69 & 76.5 \\
\hline
\end{tabular}


OH Fatigue - 2050 vs 7050 - Kt2,3, T-L, $\mathrm{RO}, 1, \mathrm{t} / 2$ - Th=75-125mm

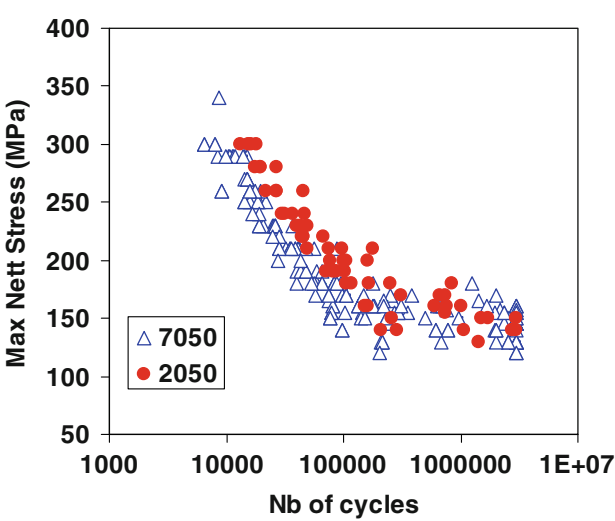

FCG Rates - 2050 vs 7050 - CCT W160, B5,

T-L, R0,1, t/2

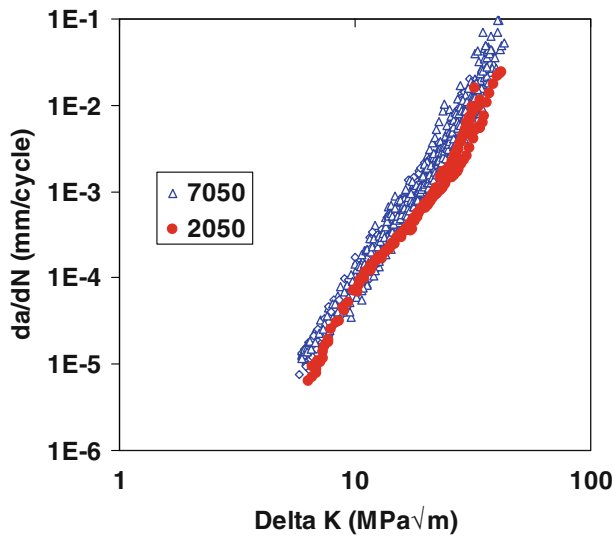

Fig. 4 Fatigue initiation and Fatigue Crack Growth (FCG) behavior of 2050 vs. 7050 plates

(a) Typical Residual Stress Profile of 2050-T84 Plates

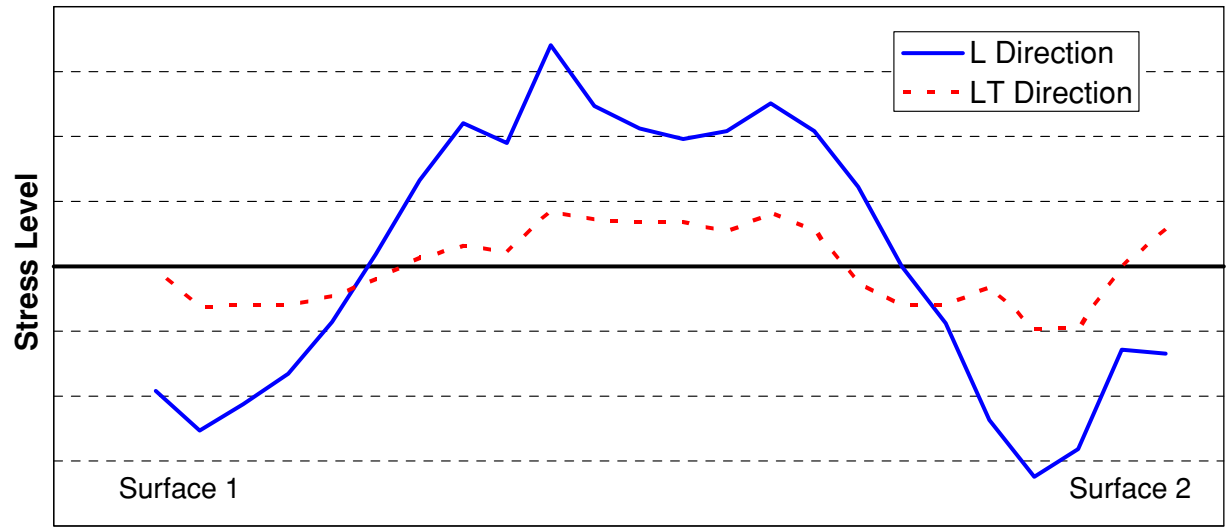

Position in thickness

(b) 2050 plate distortion for a typical stiffened structure vs offset

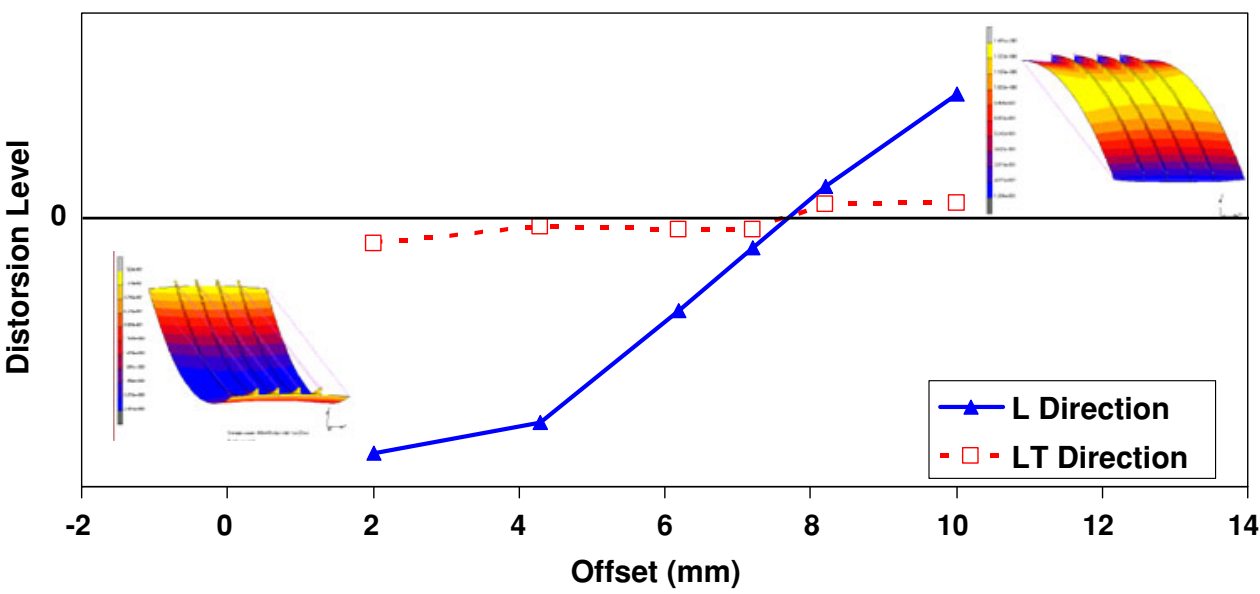

Fig. 5 Machining behavior of 2050 Plates: (a) Typical through-thickness residual stress profiles in L and LT directions. (b) Calculated distortion of a typical stiffened structure machined from a 2050 plate as a function of the part offset (position within initial plate). Illustrated FEM out-puts in the figure are magnified by a factor of 100

soft-wares able to quantify the distortion of any part machined out of a plate (Ref 9). Typical L \& LT stress profiles for 2050 are shown in Fig. 5(a). The machining behavior of a typical stiffened structure made of four integrally machined stringers; in such a case, a 2050 plate of dimensions 50 by 650 by $3500 \mathrm{~mm}$ was "virtually" (simulation) heavily machined in a 
very unsymmetrical way; part distortion was calculated using the typical stress profile of Fig. 5(a) as a function of the part position (the so-called "offset") within the initial machined plate. Results obtained are illustrated in Fig. 5(b); it is clearly identified that LT distortion is almost zero whatever the offset, which is easily explained by the low level of residual stresses in this direction. It is also seen that final part can be machined flat when accounting for the appropriate offset; in the calculated example of Fig. 5, an offset of about $8 \mathrm{~mm}$ leads to a distortion-free part.

Machining trials of different parts like frames, beams, or light gauge plates were performed at various customers and subcontractors and led to the conclusion of a similar behavior when compared to the incumbent alloy; in the cases investigated, no change in machining strategy or off-set was considered.

As mentioned above, friction stir welding can be an interesting option for reducing the buy-to-fly and thus the extra cost per kilo saved of thick 2050 plates. An illustration of possible application for wing ribs was presented during the Aeromat 2006 conference (Ref 10), where double-sided rib feet were successfully welded to a single-sided rib web. Friction stir weldability is known to be linked to the hot-workability, which proved to be fairly easy for 2050 alloy. The overall processing route had to be optimized in order to maximize strength and corrosion resistance of the weld area: this was achieved by welding on the T34 temper, and then by using a post-weld aging treatment. Good quality sound welds were achieved in this way with a typical knock-down factor of about $20 \%$ on UTS, which is better than what has been measured and reported on more difficult to weld conventional 7xxx alloys.

Another test of a longitudinal FS weldment on 2050 plates was performed in 2007 with the Institut de Soudure, located in Metz, France (Fig. 6). In such a case, two 17-m long butt-welds

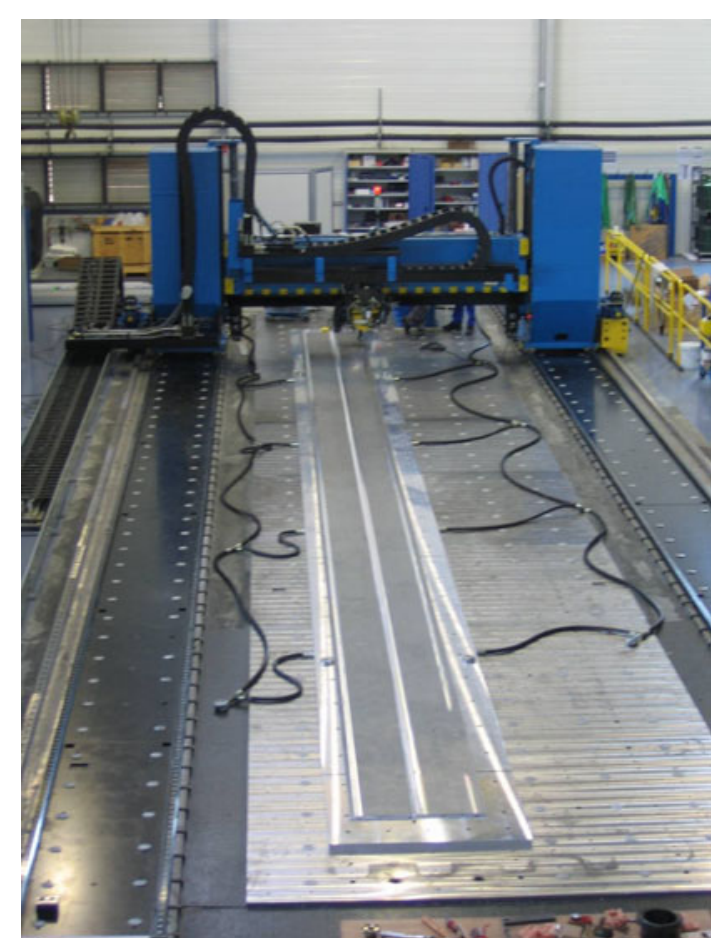

Fig. 6 Friction stir welded 17-m long panel in 2050 alloy. Trials were performed at the Institut de Soudure, Metz, France were performed on the $20 \mathrm{~mm}$ plates, using partial penetration of a stepped joint to avoid the use of a backing bar. The nonwelded area below the nugget is intended to be machined away. Optimized welding speeds of $280 \mathrm{rpm}$ and $200 \mathrm{~mm} / \mathrm{min}$ were used, for a world-first FS welding duration of about $1.5 \mathrm{~h}$.

A complete characterization of the weld area versus base metal was performed in static, damage tolerance, fatigue and corrosion (Ref 11). They confirm the good level of static knockdown factor found in the earlier studies and the fact that FSW does not degrade fatigue and damage tolerance properties of the base-metal.

Finally, it should be mentioned that 2050 Al-Cu-Li alloy can be used without any particular precaution during handling, machining, cutting, forming, etc. A Material Safety Data Sheet is available on request from the authors.

\section{Cost Reduction Exercise on 2050 Plates}

One important aspect to be considered for a widespread use of any Al-Cu-Li alloy (and this is also applicable to 2050 alloy) is the price level, which is higher than for conventional alloys of the $2 \mathrm{xxx}$ or $7 \mathrm{xxx}$ series. This higher price mostly comes from the cost of the constitutive alloying elements (in the case of 2050 alloy, mainly $\mathrm{Li}$ and $\mathrm{Ag}$ ), as well as from the special precautions to be taken during casting, where the reactive liquid metal has to be protected from outside air and moisture with special inert cover devices.

Continuous improvement activities can obviously somewhat reduce the price difference with incumbent conventional alloys, but definitely not to an extent where it can end up as cost neutral for the aircraft maker. Exchange rates of about 100 to $1000 \$$ per kilo saved have been reported and published in the past for commercial airframes (see for example Ref 12); figures even significantly higher than that were also mentioned for military and space applications. Any widespread use of 2050 alloy is thus contingent on achieving exchange rates not exceeding (and if possible significantly below) the maximum exchange rate allowed for any considered program.

For this purpose, various means to achieve the required price goals were developed; first one is based on proposing reduced buy-to-fly ratios to the customers; second one is based on developing and organizing internally and with customers appropriate recycling logistics to better recycle massive offcuts and machining swarfs.

Friction stir welding is one option to improve the utilization ratio (defined as $1-$ Weight $_{\text {scrap }} /$ Weight $_{\text {buy }}$ ) of aerospace parts, as explained above. Products with variable thickness or shapes closer to the final part can also be promoted, provided that their production costs do not compromise the cost benefit of reducing metal quantity. One has to also consider reducing all dimensional allowances generally considered for manufacturing "flexibility": this is particularly true for handing \& tooling extra widths and lengths which can represent a significant percentage of the delivered weight (and thus of the cost), depending on product dimensions.

The key role of the utilization ratio on the exchange rate of one alloy to another one more expensive is easily modeled, accounting for price difference and weight saving (Ref 4); difference in scrap values can also be introduced. This is illustrated in Fig. 7 in which all three parameters (price 


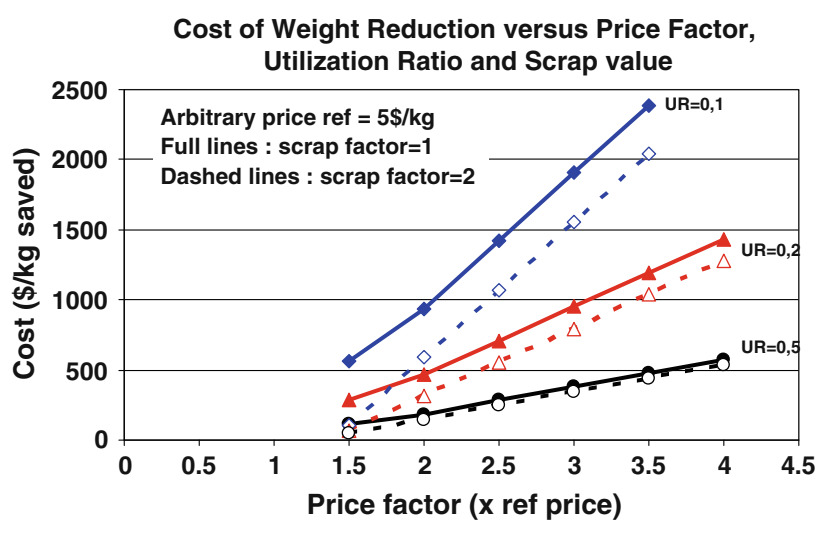

Fig. 7 Effect of price factor, utilization ratio (UR), and scrap factor (difference ratio in the scrap price) on the cost of weight saving, based on density reduction only. Price factors are expressed in multiple of the reference price. Price reference and scrap factors are arbitrary

difference, utilization ratio, and scrap factor $=$ difference ratio in the scrap price) are shown to be of great importance for the cost of weight saving. Weight saving was limited to the density benefit of 2050 versus 7050 alloy, i.e., 4.8\%. Significantly better cost figures could be obtained when accounting for the improved properties of 2050 versus 7050, like E-modulus, static yield, or fatigue.

The price factor is mainly driven by the processing costs of the proposed new alloy and by the commercial policy, and few solutions were mentioned above to increase as much as possible the utilization ratio. As far as scrap value is concerned, Alcan Aerospace has developed a policy consisting of taking back from the plants and from the OEMs (Original Equipment Manufacturer) all possible massive off-cuts and machining chips. One constraint, however, for the cost efficiency of such a recovery process is to sort the off-cuts and chips by alloy families, so that remelting is possible in the cast houses. A dedicated investment was made in the Issoire plant to be able to treat, cast as sows, and then re-melt the often contaminated machining chips. Full-scale industrial trials were performed with $\mathrm{Al}-\mathrm{Cu}-\mathrm{Li}$ chips using this dedicated equipment, and proved to be very efficient in the Li recovery, thus making it economically viable to recycle not only the massive off-cuts, but also the chips. A better scrap value can thus be proposed for 2050 versus 7050 incumbent alloy, leading to a further decrease in the cost of weight saving, as shown in Fig. 7 for hypothetical scrap factors of 1 and 2 (twice the price for the $\mathrm{Li}$ alloy).

\section{Discussion and Conclusion}

Al-Cu-Li alloy 2050 has been developed by Alcan Aerospace as an interesting low density high corrosion resistant alternative to incumbent 2024 alloy (and even to its high damage tolerant versions like 2024A and 2027) for thin plates and to reference 7050 alloy in medium-to-thick gauges. In addition, this third generation of Al-Li alloys eliminates the drawbacks of the previous generation products, with very good processability and no property deterioration over time.
2050 can thus be considered for a direct replacement of parts made of 7050 alloy, without any redesign. In such a case, the density benefit of about $5 \%$ can be directly transferred into weight benefit, meaning less fuel usage. However, when additional property improvements like higher modulus, higher static yield, better fatigue initiation, or slower crack growth rate are taken into account, it can lead to even further weight benefit of up to a total of about $10 \%$, depending on the part design drivers. The better corrosion resistance of the alloy can also bring still higher confidence to the OEM's and their customers on the use of such an Al-Cu-Li alloy.

Widespread use of this alloy depends on the economics of converting a low cost conventional alloy to a higher cost weight saving Al-Li solution. Key areas to be considered on a part-bypart basis include the increase of the material utilization ratio through whatever means (reduction of allowances, novel concepts, use of FSW which proved to be very reliable with 2050), as well as the efficient recovery of massive off-cuts and machining chips. Alcan Aerospace has developed expertise in all these fields, and is proposing a complete integrated solution which complies with the generally recognized and accepted levels of cost of weight saving.

Thanks to this complete offer, 2050 has recently entered industrial production in large quantities for various commercial aircraft and is expected to become a benchmark in the aerospace market.

\section{Acknowledgment}

The authors acknowledge the invaluable contribution of C. Sigli, B. Pitié, and B. Davo.

\section{References}

1. F.W. Gayle, W.T. Tack, F.H. Heubaum, and J.R. Pickens, High Toughness, High Strength Aluminium Alloy Design and Practice, Sixth International Aluminium-Lithium Conference, Garmisch-Partenkirchen, Germany, 7-11 Oct. 1991. 1992, p 203-208

2. W.A. Cassada, G.J. Shiflet, and E.A. Starke, The Effect of Plastic Deformation on A12CuLi (T1) Precipitation, Metall. Trans. A, 1991, 22A, p 299-306

3. B.P. Huang and Z.Q. Zheng, Effect of Li Content on Precipitation in Al-Cu-(Li)-Mg-Ag-Zr Alloys, Scripta Mater., 1998, 38, p 357-362

4. B.P. Huang and Z.Q. Zheng, Independant and Combined Roles of Trace $\mathrm{Mg}$ and $\mathrm{Ag}$ Additions in Properties Precipitation Process and Precipitation Kinetics of Al-Cu-Li-(Mg)-(Ag)-Zr-Ti Alloys, Acta Mater., 1998, 46(12), p 4381-4393

5. Z. Chen, Z. Zheng, and H. Yi, Effects of Trace Silver and Magnesium on the Microstructure and Mechanical Properties of Al-Cu-Li Alloys, J. Wuhan Univ. Technol. Mater. Sci. Ed., 2005, 20(1), p 9-12

6. G. Itoh, Q. Cui, and M. Kanno, Effects of Small Addition of Magnesium and Silver on the Precipitation of T1 Phase in an Al-4\%Cu-1.1\%Li-0.2\%Zr Alloy, Mater. Sci. Eng. A, 1996, 211, p 128-137

7. R.J. Rioja, P.E. Bretz, R.R Sawtell, W.H. Hunt, and E.A. Ludwiczak, Precipitation Reactions, Strength and Toughness of Al-Li-Cu Alloys, Aluminum Alloys: Their Physical and Mechanical Properties, Vol III, Charlottesville, Virginia, USA, 15-20 June 1986, p 1781-1797

8. F. Heymes, B. Commet, B. Dubost, Ph. Lassince, Ph. Lequeu, and G.M. Raynaud, Development of New Al Alloys for Distortion Free Machined Aluminium Aircraft Components, 1st International NonFerrous Processing \& Technology Conference, 10-12 March 1997 (St-Louis, Missouri, USA), ASM International, Materials Park, OH, 1997, p 249-255

9. Ph. Lequeu, Ph. Lassince, T. Warner, and G.M. Raynaud, Engineering for the Future: Weight Saving and Cost Reduction Initiatives, Int. J. Aircraft Eng. Aerospace Technol., 2001, 73(2), p 147-159 
10. Ph. Lequeu, R. Muzzolini, J.C. Ehrstrom, F. Bron, and R. Maziarz, High-Performance Friction Stir Welded Structures Using Advanced Alloys, Aeromat Conference, Seattle, WA, June 2006

11. Ph. Lequeu, J.C. Ehrstrom, I. Bordesoules, and Ch. Hantrais, Progress at Alcan Aerospace on the Friction Stir Welding of Al-Li
2050, 1st EUCOMAS Conference incl., CD-ROM Ort, Berlin, 26./ 27.5.2008

12. M. Mohaghegh and S.E. Axter, 31st Structures, Development of Optimum Materials for Aircraft Structures, Structural Dynamics and Materials Conference, Long Beach, CA, 2-4 April 1990 (AIAA-90-1029) 